\title{
RANDOM MATRICES, NON-BACKTRACKING WALKS, AND ORTHOGONAL POLYNOMIALS
}

\author{
SASHA SODIN
}

\begin{abstract}
Several well-known results from the random matrix theory, such as Wigner's law and the Marchenko-Pastur law, can be interpreted (and proved) in terms of non-backtracking walks on a certain graph. Orthogonal polynomials with respect to the limiting spectral measure play a rôle in this approach.
\end{abstract}

\section{INTRODUCTION}

Our goal is to explain a unified approach to the proofs of several well-known theorems in the spectral theory of random matrices and random graphs. Some of these results are formulated further in the introduction; striving to make the main idea as clear as possible, we restrict ourselves to paradigmatic examples. In particular, we only consider Bernoulli random matrices, although most proofs can be adapted to arbitrary random variables under mild assumptions on tail decay.

The method may be seen as a modification of the moment method; in the latter, used extensively since Wigner, spectral properties of a matrix $M$ are extracted from the traces $\operatorname{tr} M^{k}$ of powers of $M$. Instead, we propose to estimate $\operatorname{tr} P_{k}(M)$, where $P_{k}$ are orthogonal polynomials with respect to a certain measure $\sigma$, which is the candidate for limiting spectral measure. Perhaps surprisingly, these numbers have, in some cases, a simple combinatorial interpretation, in terms of nonbacktracking walks (see Subsection 2.3) on an appropriate graph.

One can also start from a linear recurrent relation of order two for the number of non-backtracking walks. Then a measure $\sigma$ appears from the correspondence between Jacobi (tridiagonal) matrices and measures on $\mathbb{R}$. This classical correspondence involves the orthogonal polynomials $P_{k}$ with respect to $\sigma$, that satisfy the same recurrent relation. In fact, we will see (see e.g. Lemma 2.7) that the matrix $P_{k}(M)$ is closely related to non-backtracking walks of length $k$.

Now it is natural to guess that $\sigma$ is the limiting spectral measure. We show that this is the case if the traces $\operatorname{tr} P_{k}(M)$ do not grow too

Date: February 4, 2008. 
fast; the proof is based on an analytic lemma (cf. Subsection 5.2). The combinatorial estimates (in Section 6) allow to bound these traces, for the examples that we consider.

Although orthogonal polynomials do not appear explicitly in the work of Bai and Yin on the smallest singular value of a random covariance matrix [5], the present note (as well as part of the previous work [4]) started from an attempt to understand and generalise their proof.

Similar ideas emerged also in the spectral graph theory, starting from the work of McKay [19, 20]. McKay derived an expression for the number of non-backtracking walks on a graph in terms of certain polynomials of the adjacency matrix from a certain recurrent relation and applied it to study the spectral measure of $d$-regular graphs; Friedman [8] applied it to study the spectral gap of random graphs. Li and Solé [17] noted that these are exactly the orthogonal polynomials with respect to the Kesten-McKay measure (77), and suggested to consider more general measures of the Bernstein-Szegö class (see Section 5.1). They also used the Chebyshev-Markov-Stieltjes inequalities (cf. Subsection [3.1). Related methods were developed by Brooks [7] and Serre 24].

We try to emphasise the applications to matrices other than the adjacency matrix of a graph, and especially - to random matrices.

Acknowledgement. I am grateful to my supervisor Vitali Milman for his support and useful discussions, and for urging me to write this note. The mini-courses on Random Matrix Theory taught by Leonid Pastur and Mariya Shcherbina (in Vienna and Paris) greatly improved my understanding of this field. My father helped me find the way in the literature on the problem of moments. Bo'az Klartag, Michel Ledoux, Brendan McKay, and Paul Nevai have kindly commented on a preliminary version of this note. I thank them all very much.

\subsection{Two definitions and notation.}

Definition 1.1. Let $M$ be an $n \times n$ symmetric matrix; let

$$
\lambda_{1}(M) \leq \lambda_{2}(M) \leq \cdots \leq \lambda_{n}(M)
$$

be the eigenvalues of $A$. The measure $\mu_{M}$,

$$
\mu_{M}(S)=\#\left\{1 \leq j \leq n \mid \lambda_{j}(M) \in S\right\}, \quad S \subset \mathbb{R},
$$

is called the spectral measure of $M$.

Definition 1.2. Let $\mu, \nu$ be two probability measures on $\mathbb{R}$. The Kolmogorov distance between $\mu$ and $\nu$ is defined as

$$
d_{\mathrm{K}}(\mu, \nu)=\sup _{x \in \mathbb{R}}|\mu(-\infty, x]-\nu(-\infty, x]| .
$$


Notation: Unless otherwise specified, $C, C_{1}, C_{2}, C^{\prime}, c, c^{\prime}, \cdots$ denote positive constants not depending on any parameters of the problem. Usually, upper case $C$ stand for a big constant, and lower case $c$ - for a small constant.

1.2. Symmetric random matrices. For $n \in \mathbb{N}$, let $A$ be a symmetric $n \times n$ random matrix, such that

$$
\left\{\begin{array}{l}
A_{u v} \text { are independent for } 1 \leq u \leq v \leq n, \\
\mathbb{P}\left\{A_{u v}=-1 /(2 \sqrt{n})\right\}=\mathbb{P}\left\{A_{u v}=1 /(2 \sqrt{n})\right\}=1 / 2 .
\end{array}\right.
$$

Theorem 1.3 (Wigner's law). As $n \rightarrow \infty$, the random measures $\mu_{A}$ converge (weakly, in distribution) to a deterministic measure $\sigma_{W}$ supported on $[-1,1]$;

$$
d \sigma_{W}(x)=\frac{2}{\pi} \sqrt{1-x^{2}} d x .
$$

The measure $\sigma_{\mathrm{W}}$ is called the Wigner measure.

Remark 1.4 (Precise meaning of convergence). The space $\mathcal{M}(\mathbb{R})$ of measures on $\mathbb{R}$ is equipped with the weak topology. For every $n \in \mathbb{N}$, the measure $\mu_{A}$ is a random element of $\mathcal{M}(\mathbb{R})$; its distribution is a probability measure on $\mathcal{M}(\mathbb{R})$. In Wigner's law, these distributions converge (weakly) to the distribution $\delta_{\sigma_{\mathrm{W}}}$ supported on a single point $\sigma_{\mathrm{W}} \in \mathcal{M}(\mathbb{R})$.

Theorem 1.5 (Füredi-Komlós [10]). As $n \rightarrow \infty$, the operator norm

$$
\|A\|=\max \left(\left|\lambda_{1}\right|,\left|\lambda_{n}\right|\right)
$$

of $A$ converges (in distribution) to 1.

Wigner's theorem (above) implies that

$$
\mathbb{P}\{\|A\| \leq 1-\varepsilon\} \longrightarrow 0
$$

for any $\varepsilon>0$. As for the complimentary inequality, we prove a stronger fact:

Theorem 1.6 (A. Boutet de Monvel and M. Shcherbina [6]). For some (universal) constants $c, \alpha_{1}, \alpha_{2}, \alpha_{3}>0$,

$$
\mathbb{P}\{\|A\| \geq 1+\varepsilon\} \leq \exp \left(-c n^{\alpha_{1}} \varepsilon^{\alpha_{2}}\right),
$$

provided that

$$
n^{-\alpha_{3}} \leq \varepsilon \leq 1 .
$$


1.3. Random covariance matrices. For $n \leq N$, let $B$ be an $n \times N$ random matrix (that is, $B: \mathbb{R}^{N} \rightarrow \mathbb{R}^{n}$ ), so that

$$
\left\{\begin{array}{l}
B_{u v} \text { are independent for } 1 \leq u \leq n, 1 \leq v \leq N \\
\mathbb{P}\left\{B_{u v}=-1 / \sqrt{N}\right\}=\mathbb{P}\left\{B_{u v}=1 / \sqrt{N}\right\}=1 / 2
\end{array}\right.
$$

Now we are interested in the eigenvalues

$$
0 \leq \lambda_{1} \leq \cdots \leq \lambda_{n}
$$

of the (symmetric) matrix $C=B B^{t}$.

Theorem 1.7 (Marchenko-Pastur [18]). If $n, N \longrightarrow \infty$ so that

$$
n / N \longrightarrow \xi \in(0,1],
$$

the spectral measure $\mu_{C}$ converges (weakly, in distribution) to a deterministic measure $\sigma_{M P}^{\xi}$ supported on $\left[(1-\sqrt{\xi})^{2},(1+\sqrt{\xi})^{2}\right]$;

$$
d \sigma_{M P}^{\xi}(x)=\frac{1}{2 \pi \xi x} \sqrt{\left(x-(1-\sqrt{\xi})^{2}\right)\left((1+\sqrt{\xi})^{2}-x\right)} d x .
$$

The measure $\sigma_{\mathrm{MP}}^{\xi}$ is called the Marchenko-Pastur measure.

Theorem 1.8 (Geman [11], Bai-Yin [5]). If $n, N \longrightarrow \infty$ so that

$$
n / N \longrightarrow \xi \in(0,1] \text {, }
$$

the smallest eigenvalue of $C$ converges (in distribution) to $(1-\sqrt{\xi})^{2}$, and the largest - to $(1+\sqrt{\xi})^{2}$.

Remark 1.9. The convergence of the largest eigenvalue was proved by Geman, and of the smallest - by Bai and Yin.

Similarly to the previous subsection,

$$
\mathbb{P}\left\{\lambda_{1}(C) \geq(1-\sqrt{\xi})^{2}+\varepsilon\right\} \longrightarrow 0
$$

and

$$
\mathbb{P}\left\{\lambda_{n}(C) \leq(1+\sqrt{\xi})^{2}-\varepsilon\right\} \longrightarrow 0
$$

by the Marchenko-Pastur theorem. As for the complementary inequalities, we prove the following:

Theorem 1.10 ([4]). For some (universal) constants $c, \beta_{1}, \beta_{2}, \beta_{3}>0$,

$$
\begin{aligned}
& \mathbb{P}\left\{\lambda_{1}(C) \leq(1-\sqrt{\xi})^{2}-\varepsilon\right\} \leq \exp \left(-c n^{\beta_{1}} \varepsilon^{\beta_{2}}\right), \\
& \mathbb{P}\left\{\lambda_{n}(C) \geq(1+\sqrt{\xi})^{2}+\varepsilon\right\} \leq \exp \left(-c n^{\beta_{1}} \varepsilon^{\beta_{2}}\right),
\end{aligned}
$$

provided that

$$
n^{-\beta_{3}} \leq \varepsilon \leq 1 .
$$


1.4. Adjacency matrix of a random $d$-regular graph. Fix $d \geq 3$; let $G=(V, E)$ be a random $d$-regular graph on $n$ vertices. That is, $G$ is picked uniformly from the collection of all graphs $G=(V, E)$ such that $\# V=n$ and

$$
\#\{u \in V \mid(u, v) \in E\}=d \quad \text { for every } v \in V .
$$

Let $A(G)$ be the adjacency matrix of $G$; that is,

$$
A(G)_{u v}= \begin{cases}1, & (u, v) \in E \\ 0, & \text { otherwise }\end{cases}
$$

Theorem 1.11 (McKay). The spectral measure $\mu_{A(G)}$ converges (weakly, in distribution, as $n \rightarrow \infty)$ to a deterministic measure $\sigma_{K M}$ supported on

$$
\begin{gathered}
{[-2 \sqrt{d-1}, 2 \sqrt{d-1}]} \\
d \sigma_{K M}(x)=\frac{d}{2 \pi} \frac{\sqrt{4(d-1)-x^{2}}}{d^{2}-x^{2}} d x .
\end{gathered}
$$

The measure $\sigma_{\mathrm{KM}}$ is called the Kesten-McKay measure.

1.5. A guide to the next sections. In Subsection 2.1 we introduce the general framework that unites all the problems listed above. In Subsection 2.2 we focus on an example,- the infinite $d$-regular tree,that should clarify the meaning of the Kesten-McKay measure, and also hint the main idea in the proofs of all the theorems. Lemma 2.7] in Subsection 2.3 relates the spectral properties of the matrices in study to certain combinatorial quantities.

We apply it in Subsection 3.1 to prove McKay's theorem, and in Subsection 3.2 - to prove Wigner's theorem. In Subsection 3.3 we sketch the proof of the Marchenko-Pastur theorem. The bounds on extremal eigenvalues are the subject of Section 4 .

Section 5 recalls some properties of orthogonal polynomials with respect to measures that appear in this note. In Section 6 we prove the combinatorial estimates used in the proofs of the theorems on random matrices. These two sections contain the technical results that we use elsewhere.

\section{Spectral measure: Limit theOrems}

2.1. Matrices on graphs. Let $G=(V, E)$ be a graph (with vertices $V$ and edges $E$ ). A (symmetric) $V \times V$ matrix $M$ is called a (symmetric) 
sign matrix on $G$ if

$$
M_{u v}=\left\{\begin{array}{ll} 
\pm 1, & (u, v) \in E \\
0, & (u, v) \notin E
\end{array} .\right.
$$

Example 2.1. If

$$
M_{u v}=\left\{\begin{array}{ll}
+1, & (u, v) \in E \\
0, & (u, v) \notin E
\end{array},\right.
$$

$M$ is the adjacency matrix $A(G)$ of $G$.

If the degree of every vertex is finite,- that is,

$$
\operatorname{deg}(v)=\#\{v \in V \mid(u, v) \in E\}<+\infty
$$

for every $v \in V$,- the matrix $M$ defines a symmetric operator on a dense subspace of $L_{2}(V)$. If moreover the degrees are uniformly bounded by a number $D, M$ is self-adjoint and $\|M\| \leq D$.

We are mainly interested in finite graphs $(\# V<+\infty)$; however, it will be convenient to have the definitions in this generality.

Let us recall the spectral theorem for self-adjoint operators (see Akhiezer and Glazman [2]).

Definition 2.2. A family of projectors $\left\{E_{t} \mid-D \leq t \leq+D\right\}$ is called a resolution of identity if

(1) $E_{-D}=0, E_{+D}=1$

(2) $E_{t} E_{t}^{\prime}=E_{\min \left(t, t^{\prime}\right)}$

(3) $\lim _{t \rightarrow t^{\prime}-0} E_{t}=E_{t^{\prime}}$.

For our operator $M$, there exists a resolution of identity such that all $E_{t}$ commute with $M$ and

$$
p(M)=\int_{-D}^{D} p(t) d E_{t}
$$

for any polynomial $p$.

The (operator-valued) measure $d E_{t}$ is called the spectral measure of $M$. In some important cases the (real) measure $d\left\langle E_{t} \delta_{v}, \delta_{v}\right\rangle$ does not depend on the choice of a vertex $v \in V$ (here $\delta_{v}(u)=\delta_{u v}$ for $\left.u, v \in V\right)$. In this case, we also call it the spectral measure of $M$ (more general definitions are available for $M=A(G)$; see Grigorchuk and Zuk [13] and references therein). 
2.2. Main example. Denote by $H_{d}=\left(V_{d}, E_{d}\right)$ the (infinite) $d$-regular tree $(d \geq 3)$; let $M$ be a symmetric sign matrix on $H_{d}$. According to (8),

$$
\langle p(M) f, f\rangle=\int_{-d}^{d} p(t) d\left\langle E_{t} f, f\right\rangle
$$

for any polynomial $p$ and any $f \in L_{2}\left(V_{d}\right)$, and in particular

$$
\left\langle p(M) \delta_{u}, \delta_{u}\right\rangle=\int_{-d}^{d} p(t) d\left\langle E_{t} \delta_{u}, \delta_{u}\right\rangle .
$$

Note that the measures $d\left\langle E_{t} \delta_{u}, \delta_{u}\right\rangle$ do not depend on $u$ (because of homogeneity). In fact, these measures also do not depend on $M$. The following fact is essentially due to Kesten [16]:

Proposition 2.3. The measures $d\left\langle E_{t} \delta_{u}, \delta_{u}\right\rangle$ are equal to the KestenMcKay measure $\sigma_{K M}^{d}$.

Proof. Define a sequence of polynomials

$$
\begin{gathered}
\left(p_{k}\right)_{k \in \mathbb{Z}_{+}}=\left(p_{k, d}\right)_{k \in \mathbb{Z}_{+}}, \quad \operatorname{deg} p_{k}=k: \\
\left\{\begin{array}{l}
p_{0}(t)=1, \quad p_{1}(t)=t / \sqrt{d} \\
p_{2}(t)=t^{2} / \sqrt{d(d-1)}-\sqrt{\frac{d}{d-1}}, \\
p_{k+1}(t)=t p_{k}(t) / \sqrt{d-1}-p_{k-1}(t) \quad(k=2,3, \cdots) .
\end{array}\right.
\end{gathered}
$$

\section{Lemma 2.4 .}

$$
\left\langle p_{k}(M) \delta_{u}, \delta_{u}\right\rangle=0 \quad \text { for } k=1,2,3 \cdots .
$$

As we shall see (in Lemma 2.7, from which our lemma follows), this equality expresses the fact that "there are no cycles in $H_{d}$ ". Now we need one more property of the polynomials $p_{k}$; for proof, see Remark 5.4 in Section 5 (and the discussion preceding it).

Lemma 2.5. The polynomials $p_{k}$ are orthogonal with respect to the measure $\sigma_{K M}$ :

$$
\int_{-d}^{d} p_{k}(t) p_{l}(t) d \sigma_{K M}^{d}(t)=\delta_{k l}, \quad k, l \in \mathbb{Z}_{+} .
$$

In view of (9) and Lemma 2.4,

$$
\int_{-d}^{d} p_{k}(t) d\left\langle E_{t} \delta_{u}, \delta_{u}\right\rangle=\delta_{k 0}, \quad k \in \mathbb{Z}_{+} .
$$


Therefore by Lemma 2.5,

$$
\int_{-d}^{d} p_{k}(t) d\left\langle E_{t} \delta_{u}, \delta_{u}\right\rangle=\int_{-d}^{d} p_{k}(t) d \sigma_{\mathrm{KM}}^{d}(t)
$$

for any $k \in \mathbb{Z}_{+}$, and hence

$$
\int_{-d}^{d} p(t) d\left\langle E_{t} \delta_{u}, \delta_{u}\right\rangle=\int_{-d}^{d} p(t) d \sigma_{\mathrm{KM}}^{d}(t)
$$

for any polynomial $p$.

2.3. Limit theorems for finite graphs. Let $G_{n}=\left(V_{n}, E_{n}\right)$ be a sequence of $d$-regular graphs,

$$
N_{n}=\# V_{n} \underset{n \rightarrow \infty}{\longrightarrow} \infty,
$$

and let $M_{n}$ be a symmetric sign matrix on $G_{n}$. The following questions arise:

(a) Is it true that

$$
\mu_{M_{n}} \longrightarrow \sigma_{\mathrm{KM}}^{d}
$$

for every sequence $M_{n}$ ?

(b) Does (12) hold for $M_{n}=A\left(G_{n}\right)$ ?

(c) Does (12) hold (a.s.) for a random sequence $M_{n}$ (that is, the entries of $M_{n}$ are random and independent up to the symmetry assumption,

$$
\left.\mathbb{P}\left\{M_{n, u v}=1\right\}=\mathbb{P}\left\{M_{n, u v}=-1\right\}=1 / 2, \quad(u, v) \in E ?\right)
$$

(d) Does the average spectral measure $\mathbb{E} \mu_{M_{n}}$ (with respect to the random choice of $M_{n}$ as in (c)) converge to $\sigma_{\mathrm{KM}}^{d}$ ?

It is easy to see that $(a) \Longrightarrow(b)$ and $(a) \Longrightarrow(c) \Longrightarrow(d)$. In fact, all the 4 are equivalent.

Denote by $c_{k}(G)$ the number of closed paths $\left(u_{0}, u_{1}, \cdots, u_{k}=u_{0}\right)$ in $G$, such that $\left(u_{j-1}, u_{j}\right) \in E$ for $1 \leq j \leq k$, and $u_{j} \neq u_{(j+2) \bmod k}$ for $1 \leq j \leq k$.

If the numbers $c_{k}(G)$ are small, $G$ looks locally like a tree; hence the spectral properties of matrices on $G$ should resemble those of matrices on $H_{d}$ (cf. Proposition [2.3). This is indeed the case; the following proposition generalises the result of McKay [19] on adjacency matrices (see also Serre [24]).

Proposition 2.6. For every one of the questions (a)-(d), the answer is positive iff $c_{k}\left(G_{n}\right) / N_{n} \rightarrow 0$ for $k=1,2, \cdots$. 
To prove the proposition, we need some notation. Let

$$
\mathfrak{W}_{u v}(k)=\mathfrak{W}_{u v}(k, G)=\left\{\left(u_{0}=u, u_{1}, \cdots, u_{k}=v\right) \mid\left(u_{j}, u_{j+1}\right) \in E\right\}
$$

be the collection of paths from $u$ to $v$ in $G$. Consider the subcollection

$$
\widetilde{\mathfrak{W}}_{u v}(k)=\left\{\left(u_{0}, \cdots, u_{k}\right) \in \mathfrak{W}_{u v}(k) \mid u_{j} \neq u_{j-2} \quad \text { for } \quad j \geq 2\right\}
$$

of non-backtracking paths, and the subsubcollection

$$
\widetilde{\mathfrak{W}}_{u v}^{\text {even }}(k) \subset \widetilde{\mathfrak{W}}_{u v}(k)
$$

of paths on which every edge appears an even number of times.

Finally, denote

$$
\left\{\begin{array}{ll}
\mathfrak{W}(k, G) & =\bigcup_{u \in V} \mathfrak{W}_{u u}(k, G), \\
\widetilde{\mathfrak{W}}(k, G) & =\bigcup_{u \in V} \widetilde{\mathfrak{W}}_{u u}(k, G), \\
\widetilde{\mathfrak{W}^{\text {even }}}(k, G) & =\bigcup_{u \in V} \widetilde{\mathfrak{W}}_{u u}^{\text {even }}(k, G) .
\end{array} .\right.
$$

Lemma 2.7. Let $G=(V, E)$ be a d-regular graph and let $p_{k}=p_{k, d}$ be defined as in (10).

(1) For any symmetric sign matrix $M$ on $G$, and any $u, v \in V$,

$$
p_{k}(M)_{u v}=\left\langle p_{k}(M) \delta_{u}, \delta_{v}\right\rangle=\frac{\sum^{*} M_{u_{0} u_{1}} M_{u_{1} u_{2}} \cdots M_{u_{k-1} u_{k}}}{\sqrt{d}(d-1)^{(k-1) / 2}},
$$

where the sum is over $\left(u_{0}, u_{1}, \cdots, u_{k}\right) \in \widetilde{\mathfrak{W}}_{u v}(k)$.

(2) In particular,

$$
\left|\left\langle p_{k}(M) \delta_{u}, \delta_{u}\right\rangle\right| \leq \frac{\# \widetilde{\mathfrak{W}}_{u u}(k)}{\sqrt{d}(d-1)^{(k-1) / 2}},
$$

with equality for $M= \pm A(G)$.

(3) For a randomly chosen $M$,

$$
\mathbb{E}\left\langle p_{k}(M) \delta_{u}, \delta_{u}\right\rangle=\frac{\# \widetilde{\mathfrak{W}}_{u u}^{\text {even }}(k)}{\sqrt{d}(d-1)^{(k-1) / 2}} .
$$

Proof.

(1) For $k=1$, the statement is trivial. Next,

$$
\begin{aligned}
p_{2}(M)_{u v} & =\frac{1}{\sqrt{d(d-1)}}\left(M^{2}-d \mathbf{1}\right)_{u v} \\
& = \begin{cases}\frac{1}{\sqrt{d(d-1)}} \sum_{w} M_{u w} M_{w v}, & u \neq v \\
\frac{1}{\sqrt{d(d-1)}}\left(\sum_{w} M_{u w}^{2}-d\right)=0, & u=v\end{cases}
\end{aligned}
$$


On the other hand,

$$
\widetilde{\mathfrak{W}}_{u v}(k)= \begin{cases}\{(u, w, v) \mid(u, w),(w, v) \in E\}, & u \neq v \\ \varnothing, & u=v\end{cases}
$$

therefore the right-hand side of (13) for $k=2$ is equal to the right-hand side of (16).

Now proceed by induction.

(2) Follows immediately from 1.

(3) Take the expectation of both sides of (13) and observe that if $\mathfrak{s}_{1}, \cdots, \mathfrak{s}_{\mathfrak{k}}$ are random signs drawn with replacement from a collection $\mathfrak{S}$ of independent random signs, then

$$
\mathbb{E} \mathfrak{s}_{1} \mathfrak{s}_{2} \cdots \mathfrak{s}_{\mathfrak{k}}=\left\{\begin{array}{l}
1, \quad \text { every term } \mathfrak{s} \in \mathfrak{S} \text { appears an even number } \\
0, \quad \text { of times in the product ( } 0 \text { is even!) }
\end{array}\right.
$$

Recall the following fact (cf. Feller [9, Ch. VIII, §6]):

Proposition. Let $\left(\mu_{n}\right)$ be a sequence of probability measures such that

$$
\int x^{k} d \mu_{n}(x) \longrightarrow \int x^{k} d \mu(x), \quad k=1,2,3, \cdots,
$$

where $\mu$ is a probability measure with compact support. Then

$$
\mu_{n} \longrightarrow \mu
$$

Now Proposition 2.6 follows from the next lemma:

Lemma 2.8. Let $G_{n}=\left(V_{n}, E_{n}\right)$ be a sequence of d-regular graphs,

$$
\# V_{n} \underset{n \rightarrow \infty}{\longrightarrow} \infty \text {. }
$$

The following are equivalent:

(1) For any $k \in \mathbb{N}$,

$$
\# \widetilde{\mathfrak{W}}\left(k, G_{n}\right) / \# V_{n} \longrightarrow 0
$$

as $n \rightarrow \infty$.

(2) For any $k \in \mathbb{N}$,

$$
\# \widetilde{\mathfrak{W}}^{\text {even }}\left(k, G_{n}\right) / \# V_{n} \longrightarrow 0 .
$$

(3) For any $k \in \mathbb{N}$,

$$
c_{k}\left(G_{n}\right) / \# V_{n} \longrightarrow 0 .
$$


Proof. First, $\widetilde{\mathfrak{W}^{\text {even }}}(k, G) \subset \widetilde{\mathfrak{W}}(k, G)$; hence

$$
\# \widetilde{\mathfrak{W}}^{\text {even }}(k, G) \leq \# \widetilde{\mathfrak{W}}(k, G)
$$

and $1 \Longrightarrow 2$. Similarly, $c_{k}(G) \leq \widetilde{\mathfrak{W}}^{\text {even }}(2 k, G)$ (just concatenate a closed path to itself), and so $2 \Longrightarrow 3$. Finally,

$$
\# \widetilde{\mathfrak{W}}(k, G)=c_{k}(G)+\sum_{1 \leq r<k / 2}(d-2)(d-1)^{r-1} c_{k-2 r}(G) ;
$$

therefore $3 \Longrightarrow 1$.

\section{Spectral measure: ProOfs}

3.1. McKay's theorem. Let $\left(G_{n}\right)$ be a sequence of random $d$-regular graphs: $G_{n}$ is chosen uniformly from the collection of all $d$-regular graphs on $n$ vertices; let $M_{n}$ be a symmetric sign matrix on $G_{n}$.

Proposition. For any $k \in \mathbb{N}, c_{k}\left(G_{n}\right) \longrightarrow 0$ in distribution as $n \rightarrow \infty$.

This proposition was first proved by Wormald; see also McKay, Wormald and Wysocka [21] and the discussion below.

Corollary 3.1. Let $\bar{M}_{n}$ be an $n \times n$ symmetric \pm 1 matrix, $n=1,2, \cdots$. If

$$
M_{n}=\bar{M}_{n} \bullet A\left(G_{n}\right)
$$

is the Hadamard product of $\bar{M}_{n}$ and $A\left(G_{n}\right)$,- that is,

$$
M_{n, u v}=\bar{M}_{n, u v} A\left(G_{n}\right)_{u v},-
$$

then

$$
\mu_{M_{n}} \longrightarrow \sigma_{\mathrm{KM}}
$$

weakly, in distribution, as $n \longrightarrow \infty$.

In particular (for $\bar{M}_{n, u v}=1,1 \leq u, v \leq n$ ), we recover McKay's theorem formulated in Subsection 1.4, this is very similar to the original proof in [19].

Now we aim for an estimate on the rate of convergence.

Lemma 3.2. Let $\mu$ be a probability measure on $\mathbb{R}$ such that

$$
\left|\int p_{k, d} d \mu\right| \leq \varepsilon_{k}, \quad 1 \leq k \leq 2 m-2 .
$$

Then

$$
d_{K}\left(\mu, \sigma_{K M}^{d}\right) \leq C\left(1 / m+m^{6} \sqrt{\sum \varepsilon_{k}^{2}}\right),
$$

where $C>0$ is a universal constant. 
The case $\varepsilon_{1}=\cdots=\varepsilon_{2 m-2}=0$ follows from the Chebyshev-MarkovStieltjes inequalities (cf. Akhiezer [1]); we present the proof of the general case in Subsection 5.2 (see Proposition 5.6 and Remarks 5.7 5.8).

Definition 3.3. The girth $\gamma(G)$ of a graph $G$ is the size of the smallest closed cycle in $G$. In other words,

$$
\gamma(G)=\min \left\{k \mid c_{k}(G)>0\right\} .
$$

The following proposition was proved by McKay [19] with a slightly weaker estimate, and later by Li and Solé [17] using the argument that we reproduce here.

Proposition 3.4 (McKay, Li-Solé). Let $G$ be a d-regular graph. Then

$$
d_{K}\left(\mu_{A(G)}, \sigma_{K M}^{d}\right) \leq \frac{C^{\prime}}{\gamma(G)}
$$

where $C^{\prime}>0$ is a universal constant.

Proof. By Lemma 2.7,

$$
\begin{aligned}
\int p_{k} d \mu_{A(G)} & =\sum p_{k}\left(\lambda_{i}(A(G))\right) / n \\
& =\operatorname{tr} p_{k}(A) / n=\# \widetilde{\mathfrak{W}}(k, G) /\left(n \sqrt{d}(d-1)^{(k-1) / 2}\right)=0
\end{aligned}
$$

for $1 \leq k<\gamma(G)$. Therefore by Lemma 3.2 (with all $\varepsilon_{k}$ equal to 0 )

$$
d_{\mathrm{K}}\left(\mu_{A(G)}, \sigma_{\mathrm{KM}}^{d}\right) \leq \frac{C}{\gamma(G) / 2} .
$$

Remark 3.5. Obviously, the last proposition is valid for any symmetric sign matrix $M$ on $G$.

Unfortunately, the girth of a (typical) random $d$-regular graph is $O(1)$; therefore the proposition is not applicable. To obtain a meaningful bound in McKay's theorem for random graphs, we use the full strength of Lemma 3.2, as well as the estimates on $\# \mathfrak{W}(k, G)$ that can be extracted from the work of McKay, Wormald and Wysocka [21. We omit the details that lead to

Proposition 3.6. Let $G$ be a random d-regular graph on $n$ vertices. Then

$$
d_{K}\left(\mu(A(G)), \sigma_{K}^{d}\right) \leq C \sqrt{\frac{\log d}{\log n}}
$$


with probability $1-o(1)$ (as $n \rightarrow \infty$ ), where $C>0$ is a constant independent of $d$ and $n$. Moreover, with probability $1-o(1)$,

$$
d_{K}\left(\mu(M), \sigma_{K}^{d}\right) \leq C \sqrt{\frac{\log d}{\log n}}
$$

for all sign matrices $M$ on $G$ (simultaneously).

3.2. Wigner's law. Let $A$ be a random $n \times n$ matrix, as in (2). Then

$$
A=\widetilde{A} / \sqrt{n}+D,
$$

where $\widetilde{A}$ is a random symmetric sign matrix on the complete graph $K_{n}$ (every two vertices are connected by an edge), and $D$ is a diagonal matrix,

$$
\|D\|=1 /(2 \sqrt{n}) .
$$

We will show that

$$
\mu_{A} \stackrel{(4)}{\approx} \mu_{\widetilde{A} / \sqrt{n}} \stackrel{(3)}{\approx} \mu_{\widetilde{A} / \sqrt{n-1}} \stackrel{(2)}{\approx} \widetilde{\sigma}_{\mathrm{KM}}^{n-1} \stackrel{(1)}{\approx} \sigma_{\mathrm{W}}
$$

where $\widetilde{\sigma}_{\mathrm{KM}}^{d}$ is the Kesten-McKay measure scaled to $[-1,1]$ :

$$
d \widetilde{\sigma}_{\mathrm{KM}}^{d}(x)=d \sigma_{\mathrm{KM}}^{d}(2 \sqrt{d-1} x)=\frac{2 d(d-1)}{\pi} \frac{\sqrt{1-x^{2}} d x}{d^{2}-4(d-1) x^{2}} .
$$

Step 1: Let $d \geq 3$. Then

$$
\begin{aligned}
d_{\mathrm{K}}\left(\widetilde{\sigma}_{\mathrm{KM}}^{d}, \sigma_{\mathrm{W}}\right) & \leq \int_{-1}^{1}\left|\frac{2 d(d-1)}{\pi} \frac{\sqrt{1-x^{2}}}{d^{2}-4(d-1) x^{2}}-\frac{2}{\pi} \sqrt{1-x^{2}}\right| d x \\
& =\int_{-1}^{1}\left|\frac{d(d-1)}{d^{2}-4(d-1) x^{2}}-1\right| \times \frac{2}{\pi} \sqrt{1-x^{2}} d x \\
& \leq \int_{-1}^{1} \frac{\left|d-4(d-1) x^{2}\right|}{d^{2}-4(d-1) x^{2}} \times \frac{2}{\pi} \sqrt{1-x^{2}} d x \\
& \leq \frac{3 d}{(d-2)^{2}} \leq C / d
\end{aligned}
$$

for some universal constant $C>0$.

In particular,

$$
d_{\mathrm{K}}\left(\widetilde{\sigma}_{\mathrm{KM}}^{n-1}, \sigma_{\mathrm{W}}\right) \leq C_{1} / n .
$$

Step 2: Observe that

$$
d_{\mathrm{K}}\left(\mu_{\widetilde{A} / \sqrt{n-1}}, \widetilde{\sigma}_{\mathrm{KM}}^{n-1}\right)=d_{\mathrm{K}}\left(\mu_{\widetilde{A}}, \sigma_{\mathrm{KM}}^{n-1}\right) .
$$

Now we are in the familiar setting of symmetric sign matrices on a graph. 
First consider the average spectral measure $\mathbb{E} \mu_{\widetilde{A}}$. By Lemma 3.2 ,

$$
\begin{aligned}
& d_{\mathrm{K}}\left(\mathbb{E} \mu_{\widetilde{A}}, \sigma_{\mathrm{KM}}^{n-1}\right) \\
& \quad \leq C\left(1 / m+m^{6} \sqrt{\sum_{k=1}^{2 m-2}\left[\int p_{k, n-1}(x) d \mathbb{E} \mu_{\widetilde{A}}(x)\right]^{2}}\right)
\end{aligned}
$$

we will take $m=c n^{1 / 10}$. By Lemma 2.7,

$$
\begin{aligned}
\int p_{k, n-1}(x) d \mathbb{E} \mu_{\widetilde{A}}(x) & =\sum_{u=1}^{n} \mathbb{E}\left\langle p_{k, n-1}(A) \delta_{u}, \delta_{u}\right\rangle / n \\
& =\# \widetilde{\mathfrak{W}}^{\text {even }}\left(k, K_{n}\right) /\left(n \sqrt{n-1}(n-2)^{(k-1) / 2}\right) .
\end{aligned}
$$

Obviously, $\widetilde{\mathfrak{W}}^{\text {even }}\left(k, K_{n}\right)=\varnothing$ for odd $k$, whereas for even $k$

$$
\# \widetilde{\mathfrak{W}}^{\text {even }}\left(k, K_{n}\right) \leq C k n^{k / 2} / 4 \leq C k n^{k / 2}
$$

by Proposition 6.2 (that we prove in Subsection 6.2). Hence

$$
0 \leq \int p_{k, n-1}(x) d \mathbb{E} \mu_{\widetilde{A}}(x) \leq C k / n .
$$

By (19), we have proved that

$$
d_{\mathrm{K}}\left(\mathbb{E} \mu_{\widetilde{A}}, \sigma_{\mathrm{KM}}^{n-1}\right) \leq C_{1} / n^{1 / 10}
$$

and therefore

$$
\begin{aligned}
d_{\mathrm{K}}\left(\mathbb{E} \mu_{\widetilde{A} / \sqrt{n-1}}, \sigma_{\mathrm{W}}\right) \leq d_{\mathrm{K}}\left(\mathbb{E} \mu_{\widetilde{A} / \sqrt{n-1}}, \widetilde{\sigma}_{\mathrm{KM}}^{n-1}\right)+d_{\mathrm{K}}\left(\widetilde{\sigma}_{\mathrm{KM}}^{n-1}, \sigma_{\mathrm{W}}\right) \\
=d_{\mathrm{K}}\left(\mathbb{E} \mu_{\widetilde{A}}, \sigma_{\mathrm{KM}}^{n-1}\right)+d_{\mathrm{K}}\left(\widetilde{\sigma}_{\mathrm{KM}}^{n-1}, \sigma_{\mathrm{W}}\right) \leq C_{2} / n^{1 / 10} .
\end{aligned}
$$

Steps 3 and 4: It remains to recall (18) and deduce

Proposition 3.7. There exists a universal constant $C$ such that, for a random matrix $A$ defined by (2),

$$
d_{K}\left(\mathbb{E} \mu_{A}, \sigma_{W}\right) \leq C / n^{1 / 10} .
$$

With some more effort, it is possible to prove a slightly stronger proposition:

Proposition 3.8. There exists a universal constant $C$ such that, for a random matrix $A$ defined by (2),

$$
d_{K}\left(\mu_{A}, \sigma_{W}\right) \leq C / n^{1 / 10} .
$$

with probability $1-o(1)$ (as $n \rightarrow \infty)$. 
Remark 3.9. Götze and Tikhomirov proved [14] that the left-hand sides of both (23) and (24) are not greater than $C / \sqrt{n}$; however, their argument is much more intricate.

3.3. Marchenko-Pastur law. Let $B$ be a random $n \times N$ matrix, as in (44). Define an $(n+N) \times(n+N)$ matrix $\widetilde{B}$ in the following way:

$$
\widetilde{B}=\left(\begin{array}{cc}
0 & \sqrt{N} B^{t} \\
\sqrt{N} B & 0
\end{array}\right)
$$

Then $\widetilde{B}$ is a symmetric sign matrix on the complete bipartite graph $K_{n, N}$ :

$$
\begin{aligned}
& V_{n, N}=\left\{1^{\prime}, \cdots, n^{\prime}, 1^{\prime \prime}, \cdots, N^{\prime \prime}\right\} \\
& E_{n, N}=\left\{\left(u^{\prime}, v^{\prime \prime}\right) \mid 1 \leq u \leq n, 1 \leq v \leq N\right\} .
\end{aligned}
$$

The graph $K_{n, N}$ is not regular (unless $n=N$ ); however, it is biregular (of bi-degree $(N, n)$ ).

Definition 3.10. A graph $G=\left(V^{\prime} \cup V^{\prime \prime}, E\right)$ is called bi-regular (of bi-degree $\left.\left(d^{\prime}, d^{\prime \prime}\right)\right)$ if

(1) $E \subset V^{\prime} \times V^{\prime \prime}$

(2) The degree of every vertex $v^{\prime} \in V^{\prime}$ equals $d^{\prime}$, and the degree of every vertex $v^{\prime \prime} \in V^{\prime \prime}$ equals $d^{\prime \prime}$.

Li and Solé proved [17] an analogue of Lemma 2.7 for bi-regular graphs and used it to recover the spectral measure of the bi-regular tree (first computed by Godsil and Mohar [12]), and to show that the spectral measure is not far from it for finite bi-regular graphs of large girth, and for random bi-regular graphs. Here we focus on the limiting case $n, N \longrightarrow \infty$.

Let

$$
\xi_{1}=(n-2) / N, \quad \xi_{2}=(n-1)(N-1) / N^{2} ;
$$

note that $\xi_{1}, \xi_{2} \longrightarrow \xi$ under the assumptions of the Marchenko-Pastur theorem. Define a sequence of polynomials $q_{k}=q_{k, \xi_{1}, \xi_{2}}$ :

$$
\begin{aligned}
& q_{0}(t)=1, \quad q_{1}(t)=(t-1) / \sqrt{\xi_{2}}, \\
& q_{k+1}(t)=\left(t-1-\xi_{1}\right) q_{k}(t) / \sqrt{\xi_{2}}-q_{k-1}(t) .
\end{aligned}
$$

\section{Lemma 3.11.}

(1) The polynomials $q_{k}$ are orthogonal with respect to a certain (explicit) measure $\sigma_{G M}^{n, N}$ supported on

$$
\left[1-2 \sqrt{\xi_{2}}+\xi_{1}, 1+2 \sqrt{\xi_{2}}+\xi_{1}\right] .
$$


(2) If $n, N \longrightarrow \infty$ so that $n / N \rightarrow \xi$, the measure converges weakly to the Marchenko-Pastur measure $\sigma_{M P}^{\xi}$. Moreover,

$$
d_{K}\left(\sigma_{G M}^{n, N}, \sigma_{M P}^{\xi}\right) \leq C / n
$$

Sketch of proof. Both facts can be deduced from an explicit formula for $\sigma_{G M}^{n, N}$, that follows from Bernstein-Szegö formulæ in Subsection 5.1 (cf. Li and Solé [17]).

Remark 3.12. For fixed $k$,

$$
q_{k, \xi_{1}, \xi_{2}} \longrightarrow q_{k, \xi, \xi}
$$

$q_{k, \xi, \xi}$ are orthogonal with respect to $\sigma_{\mathrm{MP}}^{\xi}$ according to Example 5.5] in Subsection 5.1. Therefore the convergence in 2 can be seen without writing the explicit formulæ for $\sigma_{G M}^{n, N}$.

The following lemma is an analogue of Lemma 2.7, the proof is analogous.

Lemma 3.13. If $M$ is an $n \times N$ matrix the entries of which are equal to \pm 1 , then

$$
q_{k}\left(M M^{t} / N\right)_{u v}=\frac{\sum^{*} M_{u_{0}^{\prime} u_{1}^{\prime \prime}} M_{u_{1}^{\prime} u_{1}^{\prime \prime}} M_{u_{1}^{\prime} u_{2}^{\prime \prime}} M_{u_{2}^{\prime} u_{2}^{\prime \prime}} \cdots M_{u_{k-1}^{\prime} u_{k}^{\prime \prime}} M_{u_{k}^{\prime} u_{k}^{\prime \prime}}}{(n N)^{k / 2}},
$$

where the sum is over

$$
\left(u_{0}^{\prime}, u_{1}^{\prime \prime}, u_{1}^{\prime}, u_{2}^{\prime \prime}, \cdots, u_{k-1}^{\prime}, u_{k}^{\prime \prime}, u_{k}^{\prime}\right) \in \widetilde{\mathfrak{W}}_{u v}\left(2 k, K_{n, N}\right) .
$$

Now,

$$
\begin{aligned}
\int q_{k} d \mathbb{E} \mu_{C} & =n^{-1} \operatorname{tr} q_{k}(C) \\
& =\sum_{u=1}^{n} \# \widetilde{\mathfrak{W}}_{u^{\prime} u^{\prime}}\left(2 k, K_{n, N}\right) /\left(n(n N)^{k / 2}\right) \\
& <\# \widetilde{\mathfrak{W}}\left(2 k, K_{n, N}\right) /\left(n(n N)^{k / 2}\right) .
\end{aligned}
$$

For $k \leq c \xi^{3 / 20} n^{1 / 10}$, the last quantity is bounded by

$$
C k / n
$$

according to Proposition 6.4.

Proceeding as in the previous subsection, with the general Proposition 5.6 (and the following remarks) instead of Lemma 3.2, we can deduce the following form of the Marchenko-Pastur theorem: 
Proposition 3.14. Under the assumptions of the Marchenko-Pastur theorem,

$$
d_{K}\left(\mathbb{E} \mu_{C}, \sigma_{M P}^{\xi}\right) \leq C /\left(\xi^{3 / 20} n^{1 / 10}\right) ;
$$

moreover,

$$
d_{K}\left(\mu_{C}, \sigma_{M P}^{\xi}\right) \leq C^{\prime} /\left(\xi^{3 / 20} n^{1 / 10}\right)
$$

with probability $1-o(1)$.

Remark 3.15. For $\xi$ bounded away from 1, Götze and Tikhomirov proved [15] a better estimate $C / n^{1 / 2}$ for the left-hand sides in these inequalities.

\section{Extremal eigenvalues}

4.1. Preliminaries. In the previous sections, the convergence of the spectral measure $\mu_{A_{n}} \longrightarrow \sigma$ followed from the convergence

$$
\int P_{k} d \mu_{A_{n}} \longrightarrow 0, \quad k=1,2,3 \ldots,
$$

where $P_{k}$ are the orthogonal polynomials with respect to $\sigma$.

To obtain convergence, we only needed (25) to hold for (every) fixed $k$. However, in some of the examples, the integral on the left-hand side of (25) is small also for $k$ growing with $n$. If this is the case (for $k$ growing fast enough), no eigenvalues of $A$ can lie far from the support of $\sigma$. We formalise this observation in this section.

Bai and Yin [5] applied a similar method (in implicit form) for random covariance matrices. In [4, exponentially decaying estimates on the probability of deviations were obtained for this case, using the method Bai and Yin and a formalism similar to that of the present note. In particular, Subsection 4.3 reproduces some of the results in [4] (correcting minor errors and misprints).

4.2. The Füredi-Komlós theorem. Let $A$ be a random matrix defined as in (21). As in the first paragraph of Subsection 3.2.

$$
A=\widetilde{A} / \sqrt{n}+D,
$$

where $\widetilde{A}$ is a random sign matrix on the complete graph $K_{n}$ and $\|D\| \leq$ $1 / 2 \sqrt{n}$. Recall the estimate (20):

$$
0 \leq \mathbb{E} \sum_{i=1}^{n} p_{k, n-1}\left(\lambda_{i}(\widetilde{A})\right) \leq C k, \quad k \leq c n^{1 / 10}
$$


By Chebyshev's inequality,

$$
\mathbb{P}\left\{\sum_{i=1}^{n} p_{k, n-1}\left(\lambda_{i}(\widetilde{A})\right) \geq L\right\} \leq C k / L, \quad L \geq 0 .
$$

Now, $p_{k, n-1}$ are orthogonal with respect to the measure $\sigma_{\mathrm{KM}}^{n-1}$ supported on $[-2 \sqrt{n-1}, 2 \sqrt{n-1}]$. Therefore, for large $k, p_{k}$ tend to infinity very fast outside this interval. More formally, we have the following

Lemma 4.1. There exists a universal constant $C>0$ such that the inequalities

(1) $\inf _{t \in \mathbb{R}} p_{k}(t) \geq-C k$;

(2) $\inf _{|t| \geq 2 \sqrt{n-1}(1+\varepsilon)} p_{k}(t) \geq \exp \left(C^{-1} k \sqrt{\varepsilon}\right)$

hold for any even $k \geq 2$ and any $0 \leq \varepsilon \leq 1$.

These estimates follow from the formulæ in Example 5.3, combined with (29).

Suppose $\widetilde{A}$ has at least one eigenvalue outside

$$
(-2 \sqrt{n-1}(1+\varepsilon), 2 \sqrt{n-1}(1+\varepsilon)), \quad \varepsilon \geq C_{2} \log ^{2} n / k^{2} .
$$

Then, by the above lemma,

$$
\sum_{i=1}^{n} p_{k, n-1}\left(\lambda_{i}(\widetilde{A})\right) \geq \exp \left(C^{-1} k \sqrt{\varepsilon}\right)-C(n-1) k \geq \exp \left(C_{1}^{-1} k \sqrt{\varepsilon}\right) .
$$

According to (26), the probability of this event is at most

$$
C k \exp \left(-C_{1}^{-1} k \sqrt{\varepsilon}\right) \leq \exp \left(-C_{3}^{-1} k \sqrt{\varepsilon}\right) .
$$

Taking $k=2\left\lfloor c n^{1 / 10} / 2\right\rfloor$ and recalling (18), we obtain the following quantitative form of the Füredi-Komlós theorem:

Theorem 4.2. Let $A$ be a random symmetric $n \times n$ matrix (as in (2)); let

$$
C \log ^{2} n / n^{1 / 5} \leq \varepsilon \leq 1
$$

Then

$$
\mathbb{P}\{\|A\| \geq 1+\varepsilon\} \leq \exp \left(-C^{-1} n^{1 / 10} \sqrt{\varepsilon}\right) ;
$$

here $C>0$ is a universal constant.

In particular, we recover Theorem 1.6 with $\alpha_{1}=1 / 10, \alpha_{2}=1 / 2$, $\alpha_{3}=0.0999$.

General concentration results yield an improvement $\alpha_{1}=1, \alpha_{2}=$ 2 ; this was brought to our attention by Michel Ledoux. The formal argument is as follows: 


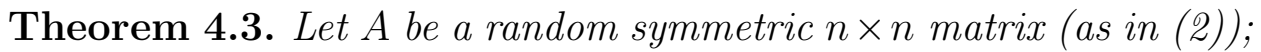
let

Then

$$
C_{1} \log ^{2} n / n^{1 / 5} \leq \varepsilon \leq 1 .
$$

here $C_{1}>0$ is a universal constant.

Proof. By (27) with $\varepsilon=C \log ^{2} n / n^{1 / 5}$, the median of $\|A\|$ is rather close to 1:

$$
\text { Med }\|A\| \leq 1+C \log ^{2} n / n^{1 / 5} .
$$

Therefore by the result of Alon, Krivelevich and $\mathrm{Vu}[3]$,

$$
\mathbb{P}\left\{\|A\| \geq 1+C \log ^{2} n / n^{1 / 5}+\varepsilon\right\} \leq 8 \exp \left(-n \varepsilon^{2} / 32\right) .
$$

Remark 4.4. The original proof of Boutet de Monvel and Shcherbina [6] yields $\alpha_{1}=1 / 2, \alpha_{2}=3 / 2, \alpha_{3}=0.333$. The estimate (28) with slightly better constants can be also deduced from a corresponding estimate for Gaussian matrices.

4.3. Bai-Yin theorem. Proceed similarly to the proof of the FürediKomlós theorem. According to Subsection 3.3

$$
\mathbb{E} \sum_{i=1}^{n} q_{k}\left(\lambda_{i}(C)\right) d \mu_{C} \leq C k
$$

for $k \leq c \xi^{3 / 20} n^{1 / 10}$; hence

$$
\mathbb{P}\left\{\sum_{i=1}^{n} q_{k}\left(\lambda_{i}(C)\right) d \mu_{C} \geq L\right\} \leq C k / L .
$$

Lemma 4.1 extends verbatim:

Lemma 4.5. There exists a universal constant $C>0$ such that the inequalities

(1) $\inf _{t \in \mathbb{R}} q_{k}(t) \geq-C k$;

(2) $\inf _{\left|t-1-\xi_{1}\right| \geq 2 \sqrt{\xi_{2}}(1+\varepsilon)} q_{k}(t) \geq \exp \left(C^{-1} k \sqrt{\varepsilon}\right)$

hold for any even $k \geq 2$ and any $0 \leq \varepsilon \leq 1$.

Now assume $C$ has at least one eigenvalue outside

$$
\left[(1-\sqrt{\xi})^{2}-\varepsilon,(1+\sqrt{\xi})^{2}+\varepsilon\right] .
$$

Then

$$
\sum_{i=1}^{n} q_{k}\left(\lambda_{i}(C)\right) d \mu_{C} \geq \exp \left(C^{-1} k \sqrt{\varepsilon / \xi}\right)-C_{1} k n \geq \exp \left(C_{2}^{-1} k \sqrt{\varepsilon / \xi}\right)
$$


if $\varepsilon \geq \frac{C_{3} \xi \log ^{2} n}{k}$. The probability of this event is at most

$$
C_{4} k \exp \left(-C_{2}^{-1} k \sqrt{\varepsilon / \xi}\right) \leq \exp \left(-C_{5}^{-1} k \sqrt{\varepsilon / \xi}\right)
$$

We have thus proved

Theorem 4.6. The probability that $C$ has eigenvalues outside

$$
\left[(1-\sqrt{\xi})^{2}-\varepsilon,(1+\sqrt{\xi})^{2}+\varepsilon\right]
$$

is at most

$$
\exp \left(-C^{-1} \xi^{-7 / 20} n^{1 / 10} \varepsilon^{1 / 2}\right)
$$

for

$$
\frac{C \xi^{7 / 20} \log ^{2} n}{n^{1 / 10}} \leq \varepsilon \leq 1
$$

In particular, we recover Theorem 1.10 with $\beta_{1}=1 / 10, \beta_{2}=1 / 2$, $\beta_{3}=0.0999$.

Remark 4.7. Similarly to the proof of Theorem 4.3, general concentration results yield an improvement $\beta_{1}=1, \beta_{2}=2$ in (6); this follows from the result of Meckes [22]. We are not familiar with a corresponding argument for (5).

\section{Bernstein-Szegő MEASURES}

5.1. Some formulæ. In this subsection we explain how to compute the orthogonal polynomials with respect to the measures we encounter. The formulæ we need follow from some more general formulæ, first proved by S. N. Bernstein and G. Szegö (see Szegö [25, Theorem 2.6]).

Recall that the Chebyshev polynomials $U_{k}(x)$ (of the second kind) are defined as

$$
U_{k}(\cos \theta)=\frac{\sin ((k+1) \theta)}{\sin \theta}, \quad k \in \mathbb{Z} .
$$

The following recurrent relation is well-known and easy to verify:

$$
2 x U_{k}(x)=U_{k+1}(x)+U_{k-1}(x) .
$$

Proposition. Let $\sigma$ be a measure supported on the segment $[-1,1]$, such that

$$
d \sigma(x)=\frac{2}{\pi \gamma^{2}} \frac{\sqrt{1-x^{2}} d x}{\left(\alpha^{2}+(1-\beta)^{2}\right)+2 \alpha(1+\beta) x+4 \beta x^{2}},
$$

where $\gamma>0$ and $\alpha, \beta \in \mathbb{R}$ are such that the denominator is strictly positive on $[-1,1]$. Then the polynomials $P_{k}(x)$,

$$
P_{k}(x)= \begin{cases}\gamma\left(U_{k}(x)+\alpha U_{k-1}(x)+\beta U_{k-2}(x)\right), & k>0 \\ \frac{\gamma}{\sqrt{1-\beta}}\left(U_{k}(x)+\alpha U_{k-1}(x)+\beta U_{k-2}(x)\right), & k=0,\end{cases}
$$


are orthogonal with respect to $\sigma$ :

$$
\int_{-1}^{1} P_{k}(x) P_{\ell}(x) d \sigma(x)=\delta_{k \ell}, \quad k, l \geq 0 .
$$

Remark 5.1. $P_{k}$ are linear combinations of $U_{k}$ and hence satisfy

$$
2 x P_{k}(x)=P_{k+1}(x)+P_{k-1}(x), \quad k=2,3, \cdots .
$$

Example 5.2. If $\alpha=\beta=0$ and $\gamma=1$, then

$$
d \sigma(x)=d \sigma_{\mathrm{W}}(x)=\frac{2}{\pi} \sqrt{1-x^{2}} d x
$$

is the Wigner measure;

$$
P_{k}(x)=U_{k}(x), \quad k=0,1,2, \cdots
$$

Example 5.3. Let $\alpha=0, \beta=-(d-1)^{-1}$, and $\gamma=\sqrt{(d-1) / d}$. Then

$$
d \sigma(x)=d \widetilde{\sigma}_{\mathrm{KM}}^{d}(x)=\frac{2 d(d-1)}{\pi} \frac{\sqrt{1-x^{2}}}{d^{2}-4(d-1) x^{2}} d x
$$

is the scaled Kesten-McKay measure;

$$
P_{k}(x)= \begin{cases}1, & k=0 \\ \sqrt{\frac{d-1}{d}} U_{k}(x)-\frac{1}{\sqrt{d(d-1)}} U_{k-2}(x), & k=1,2,3, \cdots\end{cases}
$$

Remark 5.4. Note that $p_{k, d}(x)=P_{k}(x / 2 \sqrt{d-1})$ (in view of (31) , this is easy to prove by induction). Therefore $p_{k, d}$ are orthogonal with respect to $\sigma_{\mathrm{KM}}^{d}$.

Example 5.5. If $\gamma=1, \alpha=\sqrt{y}$, and $\beta=0$, then

$$
d \sigma(x)=d \widetilde{\sigma}_{\mathrm{MP}}^{\xi}(x)=\frac{2}{\pi} \frac{\sqrt{1-x^{2}}}{(1+\xi)+2 \sqrt{\xi} x} d x
$$

is the scaled Marchenko-Pastur probability measure;

$$
P_{k}(x)= \begin{cases}1, & k=0 \\ U_{k}(x)+\sqrt{\xi} U_{k-1}(x), & k=1,2, \cdots\end{cases}
$$

Hence $q_{k, \xi, \xi}$ are orthogonal with respect to $\sigma_{\mathrm{MP}}^{\xi}$. 
5.2. A proposition in the spirit of P. L. Chebyshev, A. A. Markov and T. J. Stieltjes. Let $\sigma$ be a probability measure on $[-1,1]$; let $P_{0}, P_{1}, \cdots$ be the sequence of orthogonal polynomials with respect to $\sigma$, so that

$$
P_{k}(x)=\gamma_{k} x^{k}+\cdots, \quad \gamma_{k}>0
$$

Denote

$$
B_{k}=\max _{-1 \leq x \leq 1}\left|P_{k}(x)\right|, \quad \rho_{k}(x)=1 / \sum_{i=0}^{k} P_{i}(x)^{2}, \quad b_{k}=\max _{-1 \leq x \leq 1} \rho_{k}(x) .
$$

This section is devoted to the proof of the following proposition.

Proposition 5.6. Let $\mu$ be a probability measure on $\mathbb{R}$ such that

$$
\left|\int P_{k} d \mu\right| \leq \varepsilon_{k}, \quad 1 \leq k \leq 2 m-2
$$

Then

$$
d_{K}(\mu, \sigma) \leq 2 b_{m-1}+\left(1+m^{4} b_{m-1}^{2} B_{m}^{4}\right) \sqrt{\sum_{k=1}^{2 m-2} \varepsilon_{k}^{2}}
$$

This proposition is a "stability version" of the Chebyshev-MarkovStieltjes inequalities (that correspond to $\varepsilon_{1}=\varepsilon_{2}=\cdots=\varepsilon_{2 m-2}=0$ ). We learned some of the ideas in the proof from the work of Nevai [23].

Several well-known statements are stated further without proof; these statements are marked with an asterisk. The reader may find the proofs in the books of Akhiezer [1, Ch. III] or Szegő [25, Ch. II].

Remark 5.7. For every measure $\sigma$ that we encounter in this note (or, more formally, for probability measures in the class considered in the previous subsection),

$$
b_{m} \leq C / m \text { and } \quad B_{m} \leq C m .
$$

Therefore for these measures (32) implies

$$
d_{\mathrm{K}}(\sigma, \mu) \leq C\left(1 / m+m^{6} \sqrt{\sum \varepsilon_{i}^{2}}\right) .
$$

Remark 5.8. Taking $\sigma=\widetilde{\sigma}_{\mathrm{MK}}^{d}$ and scaling, we recover Lemma 3.2

Proof of Proposition 5.6. Let

$$
-1<\kappa_{1, m}<\kappa_{2, m}<\cdots<\kappa_{m, m}<1
$$


be the zeros of $P_{m}$. Choose $1 \leq s \leq m$ and construct two polynomials, $R$ and $S$, both of degree at most $2 m-2$ and such that

$$
\left\{\begin{array}{c}
R\left(\kappa_{1, m}\right)=\cdots=R\left(\kappa_{s, m}\right)=1 \\
R\left(\kappa_{s+1, m}\right)=\cdots=R\left(\kappa_{m, m}\right)=0 \\
R^{\prime}\left(\kappa_{1, m}\right)=\cdots=R^{\prime}\left(\kappa_{s-1, m}\right) \\
=R^{\prime}\left(\kappa_{s+1, m}\right)=\cdots=R^{\prime}\left(\kappa_{m, m}\right)=0
\end{array}\right.
$$

and

$$
\left\{\begin{array}{c}
S\left(\kappa_{1, m}\right)=\cdots=S\left(\kappa_{s-1, m}\right)=1 \\
\quad S\left(\kappa_{s, m}\right)=\cdots=S\left(\kappa_{m, m}\right)=0 \\
S^{\prime}\left(\kappa_{1, m}\right)=\cdots=S^{\prime}\left(\kappa_{s-1, m}\right) \\
=S^{\prime}\left(\kappa_{s+1, m}\right)=\cdots=S^{\prime}\left(\kappa_{m, m}\right)=0 .
\end{array}\right.
$$

Lemma* (Markov-Stieltjes). The inequalities

$$
R \geq \mathbf{1}_{\left(-\infty, \kappa_{s, m}\right]} \geq \mathbf{1}_{\left(-\infty, \kappa_{s, m}\right)} \geq S
$$

hold.

By the lemma, $\mu\left(-\infty, \kappa_{s, m}\right] \leq \int R d \mu$. Expanding $R=\sum_{k=0}^{2 m-2} a_{k} P_{k}$ (where $a_{k}=\int R P_{k} d \sigma$ ),

$$
\begin{aligned}
\int R d \mu & =\sum a_{k} \int P_{k} d \mu \\
& \leq a_{0}+\sum_{i=k}^{2 m-1}\left|a_{k}\right| \varepsilon_{k} \leq a_{0}+\sqrt{\sum_{k=1}^{2 m-2} a_{k}^{2}} \sqrt{\sum_{k=1}^{2 m-2} \varepsilon_{k}^{2}}
\end{aligned}
$$

Now,

$$
\begin{aligned}
\sqrt{\sum_{k=1}^{2 m-2} a_{k}^{2}} & \leq \sqrt{\sum_{k=0}^{2 m-2} a_{k}^{2}} \\
& =\sqrt{\int R^{2} d \sigma} \leq \sqrt{\int \mathbf{1}_{\left(-\infty, \kappa_{s, m}\right]}^{2} d \sigma}+\sqrt{\int(R-S)^{2} d \sigma}
\end{aligned}
$$

since definitely $R \leq \mathbf{1}_{\left(-\infty, \kappa_{s, m}\right]}+(R-S)$.

By (33) 34), $R-S$ is a square of some polynomial $p$ of degree $m-1$;

$$
p\left(\kappa_{t, m}\right)=\delta_{s t}, \quad 1 \leq t \leq m
$$

Therefore $p=\ell_{s, m}$ is $s$-th Lagrange interpolation polynomial of order $m$. 
Lemma 5.9. For $-1 \leq x \leq 1$,

$$
\left|\ell_{s, m}(x)\right| \leq m^{2} b_{m-1} B_{m}^{2} .
$$

Proof of Lemma 5.9. We start from an expression for $\ell_{s, m}$ that the reader may find in Szegő [25, Chapter XIV]:

$$
\ell_{s, m}(x)=\frac{\gamma_{m-1}}{\gamma_{m}} \rho_{m-1}\left(\kappa_{s, m}\right) P_{m-1}\left(\kappa_{s, m}\right) \frac{P_{m}(x)}{x-\kappa_{s, m}} .
$$

Let us estimate the terms one by one. First,

$$
\frac{\gamma_{m-1}}{\gamma_{m}}=\int_{-1}^{1} x P_{m-1}(x) P_{m}(x) d \sigma(x) \leq \sqrt{\int P_{m-1}^{2} d \sigma} \sqrt{\int P_{m}^{2} d \sigma}=1 .
$$

Then, $\rho_{m-1}\left(\kappa_{s, m}\right) \leq b_{m-1},\left|P_{m}\left(\kappa_{s, m}\right)\right| \leq B_{m}$. By the Lagrange meanvalue theorem and A.A.Markov's inequality (see for example Todd [26])

$$
\left|\frac{P_{m}(x)}{x-\kappa_{s, m}}\right| \leq \max _{-1 \leq y \leq 1}\left|P_{m}^{\prime}(y)\right| \leq m^{2} \max _{-1 \leq y \leq 1}\left|P_{m}(y)\right|=m^{2} B_{m} .
$$

The lemma is proved.

Now recall the Gauss-Jacobi quadrature formula.

Lemma* (Gauss-Jacobi quadrature). For any polynomial $q$ of degree not greater than $2 m-1$,

$$
\int p d \sigma=\sum_{i=1}^{m} \rho_{m-1}\left(\kappa_{i, m}\right) p\left(\kappa_{i, m}\right) .
$$

Applying (35+56), Lemma 5.9 and the Gauss-Jacobi quadrature, we obtain:

$$
\begin{aligned}
\mu\left(-\infty, \kappa_{s, m}\right] & \leq \int R d \sigma+\left(1+m^{4} b_{m-1}^{2} B_{m}^{4}\right) \sqrt{\sum \varepsilon_{k}^{2}} \\
& =\sum_{i=1}^{s} \rho_{m-1}\left(\kappa_{i, m}\right)+\left(1+m^{4} b_{m-1}^{2} B_{m}^{4}\right) \sqrt{\sum \varepsilon_{k}^{2}} .
\end{aligned}
$$

Similarly,

$$
\mu\left(-\infty, \kappa_{s, m}\right) \geq \sum_{i=1}^{s-1} \rho_{m-1}\left(\kappa_{i, m}\right)-\left(1+m^{4} b_{m-1}^{2} B_{m}^{4}\right) \sqrt{\sum \varepsilon_{k}^{2}} .
$$

The measure $\sigma$ satisfies the assumption (32) with $\varepsilon_{i}=0$; therefore

$$
\sum_{i=1}^{s-1} \rho_{m-1}\left(\kappa_{i, m}\right) \leq \sigma\left(-\infty, \kappa_{s, m}\right) \leq \sigma\left(-\infty, \kappa_{s, m}\right] \leq \sum_{i=1}^{s} \rho_{m-1}\left(\kappa_{i, m}\right) .
$$

The claim of the proposition follows. 


\section{COUnting NON-BACKTRACKING PATHS}

This section follows [4] (where walks on the complete bi-partite graph were considered, cf. Subsection 6.3); we have corrected minor errors and misprints.

6.1. Fragments. Let $G=(V, E)$ be a graph, and let

$$
\mathfrak{w}=\left(u^{*}, \cdots\right) \in \widetilde{\mathfrak{W}}^{\text {even }}(2 k, G) .
$$

Consider $\mathfrak{w}$ as a set of triples $\{(u, v, r) \mid 1 \leq r \leq 2 k\}$, meaning that the $r$ th edge of $\mathfrak{w}$ goes from $u \in V$ to $v \in V$.

Divide the edges into 3 classes. If $e \in \mathfrak{w}$ is the first edge to visit a vertex $v \in V$, we will write $e \in T_{1}$. More formally,

$$
T_{1}=\left\{(u, v, r) \in \mathfrak{w} \mid \forall r^{\prime}<r,\left(u^{\prime}, v^{\prime}, r^{\prime}\right) \in \mathfrak{w} \Longrightarrow v \notin\left\{u^{\prime}, v^{\prime}\right\}\right\} .
$$

The path $\mathfrak{w}$ is even, therefore for every $e \in \mathfrak{w}$ there will be another edge in $\mathfrak{w}$, coincident with $e$. Denote

$$
T_{2}=\left\{(u, v, r) \in \mathfrak{w} \mid \exists ! r^{\prime}<r,\left(u, v, r^{\prime}\right) \in T_{1} \vee\left(v, u, r^{\prime}\right) \in T_{1}\right\} .
$$

Finally, let $T_{3}=\mathfrak{w} \backslash\left(T_{1} \cup T_{2}\right)$.

A sequence of vertices $f=\left(u_{1}, \cdots, u_{\ell}\right)(\ell>1)$ is called a protofragment of $\mathfrak{w}$ if the following 3 conditions hold:

(i) for some $r$

$$
\left(u_{1}, u_{2}, r\right),\left(u_{2}, u_{3}, r+1\right), \cdots,\left(u_{\ell-1}, u_{\ell}, r+\ell-1\right) \in T_{1}
$$

(ii) for some $r^{\prime}(>r)$

$$
\begin{cases}\text { either } & \left(u_{1}, u_{2}, r^{\prime}\right),\left(u_{2}, u_{3}, r^{\prime}+1\right), \cdots,\left(u_{\ell-1}, u_{\ell}, r^{\prime}+\ell-1\right) \in T_{2} \\ \text { or } & \left(u_{\ell}, u_{\ell-1}, r^{\prime}\right), \cdots,\left(u_{3}, u_{2}, r^{\prime}+\ell-2\right),\left(u_{2}, u_{1}, r^{\prime}+\ell-1\right) \in T_{2}\end{cases}
$$

(iii) $f$ is maximal with respect to (i)-(ii).

If $f$ is a proto-fragment, $u_{1} \neq u^{*}$, we call its suffix $\bar{f}=\left(u_{2}, \cdots, u_{\ell}\right)$ a fragment of length $\ell-1$. If $u_{1}=u^{*}$, we call $f$ itself a fragment of length $\ell$. The vertices on $\mathfrak{w}$ are thereby divided into $F$ fragments.

Lemma 6.1. $F \leq 2 \# T_{3}+1$.

This inequality holds for any graph $G$, as one can easily verify.

\subsection{The complete graph.}

Proposition 6.2. There exist two constants $C, c>0$ such that, for $k \leq c n^{1 / 10}$,

$$
\# \widetilde{\mathfrak{W}}^{\text {even }}\left(2 k, K_{n}\right) \leq C k n^{k}
$$

The following lemma is obvious: 
Lemma 6.3. The number of different fragments of length $\ell$ in $K_{n}$ is not greater than $n^{\ell}$.

Proof of Proposition 6.2. First choose the number $S$ of distinct vertices on $\mathfrak{w}$. Then choose the lengths of the fragments: this can be done in $\left(\begin{array}{c}S \\ F-1\end{array}\right) \leq S^{F} / F$ ! ways. Next, choose the fragments themselves; by Lemma 6.3, this can be done in $\leq n^{S}$ ways.

There are $2^{F}$ possibilities to orient the fragments in $T_{2}$. Now glue the oriented fragments onto the path; this can be done in $(2 k-2 S+1)^{2 F}$ ways.

Every one of the remaining $2 k-2 S$ vertices coincides with one of the $S$ vertices on the fragments. Therefore there are $\leq S^{2 k-2 S}$ possibilities to arrange these vertices.

Therefore

$$
\begin{aligned}
\# \widetilde{\mathfrak{W}}^{\text {even }}\left(2 k, K_{n}\right) & \leq \sum_{S, F} \frac{S^{F}}{F !} n^{S} 2^{F}(2 k-2 S+1)^{2 F} S^{2 k-2 S} \\
& \leq n^{k} \sum_{S, F}\left\{\frac{C S(k-S)^{2}}{F}\right\}^{F}\left(\frac{S^{2}}{n}\right)^{k-S} .
\end{aligned}
$$

Now, $F \leq 2 \# T_{3}+1=4 k-4 S+5$; the function $x \mapsto(y / x)^{x}$ is increasing on $[0, y / e]$; therefore

$$
\begin{aligned}
\# \widetilde{\mathfrak{W}}^{\text {even }}\left(2 k, K_{n}\right) & \leq n^{k} \sum_{S, F}\left(C_{1} S(k-S)\right)^{4(k-S)}\left(\frac{S^{2}}{n}\right)^{k-S} \\
& \leq n^{k} \sum_{S, F}\left(\frac{C_{1} S^{6}(k-S)^{4}}{n}\right)^{k-S} \leq C k n^{k}
\end{aligned}
$$

for $k \leq c n^{1 / 10}$.

\subsection{The complete bipartite graph.}

Proposition 6.4. There exists two constants $C, c>0$ such that, for $k \leq c \xi^{3 / 20} n^{1 / 10}$,

$$
\# \widetilde{\mathfrak{W}}^{\text {even }}\left(2 k, K_{n, N}\right) \leq C k(n N)^{k / 2} .
$$

The following obvious lemma replaces Lemma 6.3.

Lemma 6.5. The number of different fragments of length $\ell$ in $K_{n, N}$ is not greater than

$$
2 \sqrt{N / n}(n N)^{\ell / 2} .
$$


Proof of Proposition 6.4. Similarly to the proof of Proposition 6.2.

$$
\begin{aligned}
& \# \widetilde{\mathfrak{W}}^{\text {even }}\left(2 k, K_{n, N}\right) \\
& \leq \sum_{S, F} \frac{S^{F}}{F !}(2 \sqrt{N / n})^{F}(n N)^{S / 2} 2^{F}(2 k-2 S+1)^{2 F} S^{2 k-2 S} \\
& \leq(n N)^{k / 2} \sum_{S, F}\left(\frac{C S(k-S)^{2}}{\sqrt{\xi} F}\right)^{F}\left(\frac{S^{2}}{\sqrt{n N}}\right)^{k-S} \\
& \leq(n N)^{k / 2} \sum_{S, F}\left(\frac{C_{1} S(k-S)}{\sqrt{\xi}}\right)^{4(k-S)}\left(\frac{S^{2}}{\sqrt{n N}}\right)^{k-S} \\
& \leq(n N)^{k / 2} \sum_{S, F}\left(\frac{\left.C_{1} S^{6}(k-S)^{4}\right)}{\xi^{3 / 2} n}\right)^{4(k-S)} \leq C_{2} k(n N)^{k / 2}
\end{aligned}
$$

if $k \leq c \xi^{3 / 20} n^{1 / 10}$.

\section{REFERENCES}

[1] N. I. Akhiezer, The classical moment problem and some related questions in analysis, Hafner Publishing Co., New York $1965 \mathrm{x}+253$ pp.

[2] N. I. Akhiezer, I. M. Glazman, Theory of linear operators in Hilbert space, Translated from the Russian and with a preface by Merlynd Nestell, Reprint of the 1961 and 1963 translations, Dover Publications, Inc., New York, 1993. xiv $+147+\mathrm{iv}+218 \mathrm{pp}$.

[3] N. Alon, M. Krivelevich, V. H. Vu, On the concentration of eigenvalues of random symmetric matrices, Israel J. Math. 131 (2002), 259-267

[4] Sh. Artstein-Avidan, O. Friedland, V. Milman, S. Sodin, Polynomial bounds for large Bernoulli sections of $\ell_{1}^{N}$, Israel J. Math., Vol. 156, pp. 141-156

[5] Z. D. Bai, Y. Q. Yin, Limit of the smallest eigenvalue of a large dimensional sample covariance matrix, Ann. Probab. 21 (1993), no. 3, 1275-1294

[6] A. Boutet de Monvel, M. V. Shcherbina, On the norm of random matrices (Russian), Mat. Zametki 57 (1995), no. 5, 688-698, 797; translation in Math. Notes 57 (1995), no. 5-6, 475-484

[7] R. Brooks, The spectral geometry of k-regular graphs, J. Anal. Math. 57 (1991), $120-151$

[8] J. Friedman, A Proof of Alon's Second Eigenvalue Conjecture and Related Problems, accepted to the Memoirs of the A. M. S.

[9] W. Feller, An introduction to probability theory and its applications, Vol. II, Second edition, John Wiley \& Sons, Inc., New York-London-Sydney, 1971, xxiv+669 pp.

[10] Z. Füredi, J. Komlós, The eigenvalues of random symmetric matrices, Combinatorica 1 (1981), no. 3, 233-241.

[11] S. Geman, A limit theorem for the norm of random matrices, Ann. Probab. 8 (1980), no. 2, 252-261. 
[12] C. D. Godsil, B. Mohar, Walk generating functions and spectral measures of infinite graphs, Proceedings of the Victoria Conference on Combinatorial Matrix Analysis (Victoria, BC, 1987), Linear Algebra Appl. 107 (1988), 191-206.

[13] R. I. Grigorchuk, A. Żuk, The Ihara zeta function of infinite graphs, the KNS spectral measure and integrable maps, Random walks and geometry, 141180, Walter de Gruyter GmbH \& Co. KG, Berlin, 2004

[14] F. Götze, A. Tikhomirov, Rate of convergence to the semi-circular law, Probab. Theory Related Fields 127 (2003), no. 2, 228-276

[15] F. Götze, A. Tikhomirov, Rate of convergence in probability to the MarchenkoPastur law, Bernoulli 10 (2004), no. 3, 503-548.

[16] H. Kesten, Symmetric random walks on groups, Trans. Amer. Math. Soc. 92, 1959, 336-354

[17] W.-Ch. W. Li, P. Solé, Spectra of regular graphs and hypergraphs and orthogonal polynomials, European J. Combin. 17 (1996), no. 5, 461-477

[18] V. A. Marchenko, L. A. Pastur, Distribution of eigenvalues in certain sets of random matrices (Russian), Mat. Sb. (N.S.) 72 (114), 1967, 507-536

[19] B. D. McKay, The expected eigenvalue distribution of a random labelled regular graph, Linear Algebra and its Applications, 40 (1981) 203-216

[20] B. D. McKay, Spanning trees in regular graphs, European J. Combin. 4 (1983), no. $2,149-160$.

[21] B. D. McKay, N. C. Wormald, B. Wysocka, Short cycles in random regular graphs, Electron. J. Combin. 11 (2004), no. 1, Research Paper 66, 12 pp.

[22] M. W. Meckes, Concentration of norms and eigenvalues of random matrices, J. Funct. Anal. 211 (2004), no. 2, 508-524.

[23] P. Nevai, Mean convergence of Lagrange interpolation. III, Trans. Amer. Math. Soc. 282 (1984), no. 2, 669-698

[24] J.-P. Serre, Répartition asymptotique des valeurs propres de l'opérateur de Hecke $T_{p}$, J. Amer. Math. Soc. 10 (1997), no. 1, 75-102

[25] G. Szegő, Orthogonal polynomials, American Mathematical Society, Providence, R.I., 1975. xiii+432 pp.

[26] J. Todd, Introduction to the constructive theory of functions, Academic Press, Inc., New York 1963127 pp.

School of Mathematics, Raymond and Beverly Sackler Faculty of Exact Sciences, Tel Aviv University, Tel Aviv, 69978, Israel.

E-mail address: sodinale@tau.ac.il 